Classification

Physics Abstracts

$47.15-61.40 \mathrm{~K}-81.15 \mathrm{~L}$

\title{
Spreading laws for liquid polymer droplets : interpretation of the « foot »
}

\author{
F. Brochard and P. G. de Gennes \\ Collège de France, 75231 Paris Cedex 05, France
}

(Reçu le 27 mars 1984, accepté le 20 avril 1984)

\begin{abstract}
Résumé. - Une goutte de polymère fondu s'étale, sur certaines surfaces, en montrant un " pied » macroscopique, qui précède le corps de la goutte. Nous expliquons ce " pied " par un effet de glissement du polymère sur la surface solide : l'existence d'une grande longueur d'extrapolation $b$, pour le champ de vitesses, dans de tels cas, a déjà été prévue par l'un d'entre nous. Nous calculons la forme détaillée du pied (cote $\zeta$ fonction de la distance au bord $x: \zeta(x) \sim x^{3 / 2}$ ) et montrons que la hauteur du pied est comparable à la longueur d'extrapolation $b$. Celle-ci peut être très grande ( $>100$ microns) pour des polymères enchevêtrés sur une surface lisse. Par contre, si la surface est rugueuse, ou si certaines chaînes sont ancrées au solide, la longueur $b$ redevient très petite : ceci explique que l'existence du « pied » soit très sensible à l'état de la surface solide.
\end{abstract}

\begin{abstract}
Depending upon the nature of the polymer-substrate system, it has been observed that the shape of a spreading polymer droplet on a flat surface could be either a spherical cap - as for low viscosity liquids - or a spherical cap with a projecting macroscopic « foot ». We interpret this " foot " by an effect of finite slip of the polymer liquid at the surface : the existence of a large extrapolation length $(b)$ for the velocity field of polymeric liquids near a smooth surface was predicted long ago by one of us. We calculate the detailed shape of the foot, and show that the foot thickness is comparable to $b$; this length may reach very high values ( $>100$ microns) for entangled polymers. On the other hand, if the surface is rough, or if some chains are strongly bound to it, $b$ is drastically reduced : this explains why the existence of the « foot » depends on the precise conditions at the surface.
\end{abstract}

\section{Introduction.}

1.1. - The spreading of low viscosity liquid droplets on clean solid surfaces is now reasonably well understood.

a) If the liquid wets partially the solid (finite contact angle $\theta_{\mathrm{e}}$ ) the droplet relaxes rapidly to equilibrium.

If $R_{\mathrm{F}}$ is the equilibrium radius, the equilibrium time is $\tau \sim R_{\mathrm{F}} / V^{*}$, where $V^{*}=\gamma / \eta$ is the "spreading velocity" resulting from a balance between surface tensions $(\gamma=$ liquid/vapour surface tension) and viscous resistance $\left(\eta=\right.$ viscosity of the liquid). For $\gamma=100, \eta=10^{-2}$, $R_{\mathrm{F}}=0.1$ (CGS), one expects $\tau=10^{-5} \mathrm{~s}$.

b) The most interesting case corresponds to perfect wetting $\left(\theta_{\mathrm{e}}=0\right)$, and we shall only consider this case. The spreading times are then very long. 
i) The macroscopic shape of the droplet is a spherical cap, with an apparent contact angle $\theta_{\mathrm{a}}(t)$ (Fig. 1).

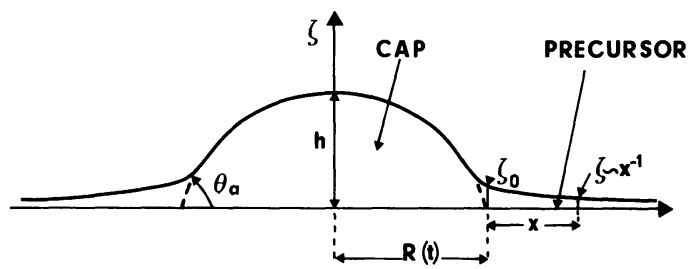

Fig. 1. - Spreading of a low viscosity liquid, assuming complete wetting (equilibrium contact angle $\theta_{\mathrm{e}} \equiv 0$ ). The " spherical cap " is dominated by capillary and viscous forces, while the precursor film is dominated by Van der Waals and viscous forces.

By optical observations of spreading oil drops, Tanner [1] finds $\theta_{\mathrm{a}}(t) \sim t^{-0.30}$. Data on the macroscopic droplet radius by Lelah and Marmur [2] are consistent with Tanner's law : They find $R(t) \sim t^{0.1}$. If $h$ is the thickness of the droplet, $\theta_{\mathrm{a}} \sim \frac{h}{R} \sim \frac{\Omega}{R^{3}}$ where $\Omega$ is the fluid volume. Then $\theta_{\mathrm{a}}(t) R^{3}(t)=$ const.

The $\theta_{\mathrm{a}}(t)$ law is well explained theoretically $[1,3]$ in terms of surface tensions and viscous forces.

ii) A microscopic precursor film advances ahead of the main droplet. This was discovered a long time ago by $\mathbf{W}$. Hardy and analysed more recently by various methods : ellipsometry and interferometry [4], scanning electron microscopy [5] and electrical resistivity [6]. The thickness is of the order of $1000 \AA$ and decreases slowly with the distance $(x)$ to the triple line.

Recently, Teletzke, Davis, and Scriven $\left({ }^{1}\right)$, and one of us [3] have interpreted this film in terms of the long range Van der Waals forces (VW) coupling the solid and the liquid. The kinetics of the spreading is controlled by VW interactions (instead of Laplace pressure), and viscous resistance. The theoretical prediction for the film thickness $\zeta(x)$ is a slowly decreasing function $\zeta(x) \sim x^{-1}$. At the junction between the film and the spherical cap, the thickness is predicted to be $\zeta_{0} \cong$ $a \theta_{\mathrm{a}}^{-1}(t)$ where $a$ is an atomic size. This explains why the precursor film is observed only with wetting liquids $\left(\theta_{a} \rightarrow 0\right)$.

1.2 SPREADING OF POLYMER LIQUIDS. - This process is technically important (paints, cosmetics, adhesives, ...). Two anomalies have been quoted :

a) in some cases the drop spreads faster than expected from the Tanner analysis : the apparent viscosity is low [7];

b) for certain substrates, the drop displays two macroscopic regions : a spherical cap, preceded by a "foot", which is visible at low magnification, and much thicker than the precursor films discussed above $[8,9]$.

1.3 AnOmalous SLIP IN POLYMER Flow NEAR A WALl. - The basic idea is described in reference [10] : consider a simple shear flow described by a velocity field $v_{x}(z) \equiv v(z)$ near a wall (at $z=0$ ). The stress at the wall $\sigma$ is proportional to the local velocity $v(0)$. The essential point is that the coefficient $k=\frac{\sigma}{v(0)}$ is independent of the molecular weight $M$ of the polymer : the entanglemeni effects responsible for the bulk viscosity $\eta(M)$ have no counterpart on a smooth surface. Thus we may write $k=\eta_{0} / a$ where $\eta_{0}$ is comparable to the viscosity of the monomer

( $\left.{ }^{1}\right)$ Unpublished. 
liquid. Far from the wall we may also write $\sigma=\eta \frac{\mathrm{d} v}{\mathrm{~d} z}$; comparing the two forms of $\sigma$ we see that the boundary conditions on the wall are :

$$
v(0)=\left.b \frac{\mathrm{d} v}{\mathrm{~d} z}\right|_{0}
$$

where $b$ is the extrapolation length

$$
b=\frac{\eta}{k}=\frac{\eta}{\eta_{0}} a
$$

a) For entangled polymer chains (degree of polymerization $N>N_{\mathrm{e}}(\sim 100)$ ), $\eta$ increases extremely rapidly with $N$. In the reptation model [11], $\eta \sim \eta_{0} N^{3} N_{\mathrm{e}}^{-2}$. This leads to $b \sim N^{3} \times$ $N_{\mathrm{e}}^{-2} a$. For $N=10^{3}, N_{\mathrm{e}}=10^{2}, a=1 \AA, b \sim 10$ microns.

b) For non entangled chains $\left(N<N_{\mathrm{e}}\right) \eta$ increases more or less linearly with $N\left(\eta \sim \eta_{0} N\right)$. No detailed analysis of the velocity field near the wall is available. If equation (4) still holds, we would get $b=N a$, i.e. $b \sim 1000 \AA$ for $N=10^{3}$.

The notion of a finite length $b$ has been introduced very early in discussions of spreading kinetics [12-14]. In some cases it was attributed to surface roughness [15], while in other cases it was related to some microscopic molecular process [16]. As explained in reference [10] we believe that slip is in fact irrelevant for most smooth surfaces (where Van der Waals forces dominate the precursor structure). But, in the special case of polymer melts, $b$ as predicted by equation (5) may become exceptionally large, and relevant.

\section{Droplet profiles.}

Let us assume that the droplet height $h$ is larger than $b$. More precisely we consider the case $h \gg b \gg \zeta_{0}$. We then expect to find three regions.

a) a spherical cap corresponding to the region where the thickness $\zeta(r)$ is larger than $b:$ here normal viscous flow takes place, the slip is negligible and we recover the Tanner laws $[1,3]$;

b) $a$ foot, where $b>\zeta(r)>\zeta_{0}$ : in the foot we have plug flows driven by the capillary pressures ;

c) a precursor film $\left(\zeta(r)<\zeta_{0}\right)$ with a plug flow driven by Van der Waals forces.

2.1 PoISEUILle OR PLUG Flows. - We restrict our attention to the limit of flat droplets $(h \ll R)$, treated in the lubrication approximation. The mean velocity $\mathrm{V}(x, y, t)$ is related to the two-dimensional pressure gradient $\nabla p$ by the equation

$$
\mathbf{V}=-\nabla p \frac{\zeta}{\eta}\left[b+\frac{1}{3} \zeta\right]
$$

In the limit $\zeta>b$ this reduces to a standard Poiseuille formula (for flow between a solid and a free surface). In the limit $\zeta<b$ equation (3) describes a plug flow, dominated by friction at the solid surface. We must add to (3) the conservation equation

$$
\frac{\partial \zeta}{\partial t}+\operatorname{div}(\zeta \mathbf{V})=0
$$

2.2 MACROSCOPIC REGIMES. - Here, the pressure gradient is provided by the surface tension :

$$
\nabla p=-\gamma \nabla\left(\nabla^{2} \zeta\right)
$$


The profile $\zeta(x, y, t)$ is then governed by

$$
\mathbf{V}=V^{*}\left(b+\frac{1}{3} \zeta\right) \zeta \nabla\left(\nabla^{2} \zeta\right)
$$

We set $\gamma / \eta=V^{*}, \gamma / \eta_{0}=V_{0}^{*}$.

2.2.1 Profile near the triple line : Foot. - We call $U=\frac{\mathrm{d} R}{\mathrm{~d} t}$ the velocity of the edge of the drop $(R(t)$ is the macroscopic observable radius). We assume that the mean velocity $V$ in the vicinity of the " triple line » can be approximated by $U$, and taken as constant. This will turn out to be correct if the foot region is smaller than the cap region. For $\zeta<b$, equation (3) can be written (in a one-dimensional form) :

$$
U=V^{*} b \zeta(x) \zeta^{\prime \prime \prime}(x)
$$

where $x$ is the distance from the edge of the drop, and where $\zeta^{\prime \prime \prime} \equiv \frac{\mathrm{d}^{3} \zeta}{\mathrm{d} x^{3}}$.

A special solution is

$$
\zeta=\left(\frac{8|x|^{3}}{3 \lambda}\right)^{1 / 2}
$$

where $\lambda=b V^{*} / U$.

For $\zeta>b$, we return to the usual spherical cap profile.

Setting $\zeta=b$ in equation (8) gives the width $f$ of the foot (represented on Fig. 2) :

$$
f=\left(\frac{3 b^{2} \lambda}{8}\right)^{1 / 3} \text {. }
$$

Typically, for $b=100 \mu$ and $U / V^{*}=10^{-3}, f \sim 1 \mathrm{~mm}$. Our calculation holds provided that $f \ll R(t)$.

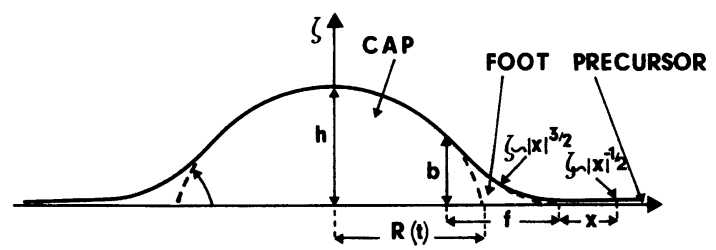

Fig. 2. - Spreading of a polymer melt $\left(\theta_{\mathrm{e}}=0\right)$. Inside both the "foot" and the "precursor" the fluid slips on the solid surface. In the « foot» the motion is dominated by capillary and friction forces.

2.2.2 Spherical cap regime $(\zeta>b)$. - For $\zeta>b$, we recover the classical case of low viscosity fluids $[1,3]$. At $\zeta=b$, the apparent contact angle deduced from (8) is $\theta_{\mathrm{a}} \cong b / f \cong\left(U / V^{*}\right)^{1 / 3}$.

This leads to the Tanner spreading law [1]

$$
U \equiv \frac{\mathrm{d} R}{\mathrm{~d} t} \cong V^{*} \theta_{\mathrm{a}}^{3}(t)
$$

For the spherical cap, $\theta_{\mathrm{a}} \cong h / R \cong \Omega / R^{3}$.

Then $R^{10}(t) \cong \Omega^{3} V^{*} t$. 
Conclusion: The macroscopic radius $R(t)$ should follow the usual spreading law $R(t) \sim t^{0.1}$; the spreading velocity is small, because of the high viscosity of polymer fluids. The new effect is the apparition of the " foot " of height $b$ and width $f \cong b \theta_{\mathrm{a}}^{-1}(t)$.

2.3 MiCROSCOPIC REGIME. - For small thickness $\zeta$, we must include the pressure provided by Van der Waals forces [7] : if $A$ is the Hamaker constant (in the non retarded regime) :

$$
\nabla p=\frac{A}{2 \pi \zeta^{4}} \nabla \zeta-\gamma \nabla \nabla^{2} \zeta
$$

The cross-over between the "foot " and the film is deduced from equations (11) and (3), used in the plug flow limit.

Taking $V=U=$ const., treating (11) as one dimensional, and setting $\zeta=\bar{z} \zeta_{0}, x=\bar{x} x_{0}$ we obtain the $a$-dimensional equation :

$$
\bar{z}^{\prime \prime \prime} \bar{z}-\bar{z}^{\prime} z^{-3}=1 \text {. }
$$

Setting $a^{2}=A / 2 \pi \gamma$ we then find that we must choose :

$$
\begin{aligned}
& \zeta_{0}=a\left(\frac{V_{0}^{*}}{U}\right)^{1 / 4}=\left(\frac{a^{3} b}{\theta_{\mathrm{a}}}\right)^{1 / 4} \\
& x_{0}=a\left(\frac{V_{0}^{*}}{U}\right)^{1 / 2}=\left(\frac{a b}{\theta_{\mathrm{a}}^{3}}\right)^{1 / 2}
\end{aligned}
$$

For $\zeta \ll \zeta_{0}$, the profile is dominated by Van der Waals forces. Again neglecting the variation of $V$ in the precursor region $(V \rightarrow U)$, the profile is given by

$$
\eta U / b=-\frac{A \zeta^{\prime}}{2 \pi \zeta^{3}}
$$

with $\eta / b=\eta_{0} / a=k$. Then

$$
\zeta^{2}(x)=\frac{2 \pi k}{A U} \frac{1}{x+x_{1}} .
$$

The profile in the film is thus very slowly decreasing. The constant $x_{1}$ is comparable to $x_{0}$.

\section{Conclusions.}

1) The existence of a macroscopic foot on a spreading droplet is a natural consequence of the predictions of reference [10] : polymer liquids can have very unexpected plug flows on a macroscopic scale. The observed foot structure [4] is often much more complex than predicted by equation (8). Note however that our discussion reduced the polymer to a Newtonian fluid : viscoelastic lags may well complicate the early stages of spreading.

2) The size of the foot is controlled by the extrapolation length $b$, and should thus be very sensitive to the microscopic properties of the solid surface : this is discussed at length in [10]. But systematic studies on foot dimensions versus polymer viscosity in the entangled regime should be carried out in the future.

3) Our analysis assumed a relatively thick droplet $(h>b)$. In the opposite limit $(h<b$ but $h \gg \zeta_{0}$ ) the spherical cap region merges with the foot. We do not know the detailed profile for this 
case. But a simple scaling discussion of equations $(3,5)$, in the limit $\zeta<b$, suggests a modified spreading law : $R(t) \sim t^{1 / 8}$.

4) For short chains $\left(N<N_{\mathrm{e}}\right)$, a more precise calculation of the extrapolation length $b$ is required : we hope to construct this in the future.

5) Note that our whole discussion is strictly restricted to pure liquids : impurities can complicate the profiles enormously - either by adsorption on the solid, or by evaporation and the resulting Marangoni effects [4].

\section{References}

[1] Tanner, L. H., J. Phys. D. 12 (1979) 1473. See also the basic work of Huh, C., Scriven, L., J. Colloid Interface Sci. 35 (1971) 85.

[2] Lelah, M. D., Marmur, A., J. Colloid Interface Sci. 82 (1981) 518.

Marmur, A., Adv. Colloid Interface Sci. 19 (1983) 75.

[3] De Gennes, P. G., C. R. Hebd. Séan. Acad. Sci. Ser. B 298 (1984) 111.

[4] Bascom, W. D., Cottington, R. L., Singleterry, C. R., Adv. Chem. Ser. 43 (1964) 355.

[5] Radigan, W., Ghiradella, H., Frisch, H. T., Schonhorn, H., Kwei, T. K., J. Colloid Interface Sci. 49 (1974) 241.

[6] Ghiradella, H., Radigan, W., Frisch, H., J. Colloid Interface Sci. 51 (1978) 522.

[7] SAwicki, G. C., in Wetting, Spreading and Adhesion, J. Padday, ed. (Academic Press (N. 7)) 1978, p. 361 .

[8] Dettre, R. M., Johnson, R. E., J. Adhes. 2 (1970) 61.

[9] Arslamov, V. V., Ivanova, T. J., OJar, V. A., Dokl. Phys. Chem. 198 (1971) 502.

[10] De Gennes, P. G., C. R. Hebd. Séan. Acad. Sci. Ser. B. 288 (1979) 219.

[11] De Gennes, P. G., Leger, L., Ann. Rev. Phys. Chem. 33 (1982) 49.

[12] Huh, C., Mason, S., J. Fluid Mech. 81 (1977) 401.

[13] Levine, S., Lowndes, J., Watson, E., Neale, G., J. Colloid Interface Sci. 73 (1980) 136.

[14] Dussanv, E., J. Fluid Mech. 77 (1976) 665.

[15] Hocking, L., J. Fluid Mech. 76 (1976) 801.

[16] Neodgi, P., Miller, C., J. Colloid Interface Sci. 86 (1982) 525. 